\title{
Nonlinear Acoustics in Diatomic Gases Using Direct Simulation Monte Carlo
}

\author{
Amanda L. Danforth", and Lyle N. Long ${ }^{\dagger}$ \\ ${ }^{*}$ Graduate Program in Acoustics, Pennsylvania State University, University Park, PA, 16802, USA \\ ${ }^{\dagger}$ Aerospace Engineering Department, Pennsylvania State University, University Park, PA, 16802, USA
}

\begin{abstract}
The Direct Simulation Monte Carlo (DSMC) method has been very successful for the study of many problems in rarefied gas dynamics and hypersonic flow. The extension to applications such as acoustics will provide a useful tool for capturing all physical properties of interest for nonlinear acoustic problems, such as dispersion, attenuation, harmonic generation and nonequilibrium effects. The validity of DSMC for the entire range of Knudsen numbers $(K n)$, where $K n$ is defined as the mean free path divided by the wavelength, allows for the exploration of sound propagation at low $K n$ (low frequency, atmospheric conditions) as well as sound propagation at high $K n$ (high frequency, dilute gases, or in microdevices). This paper will present the application of DSMC to nonlinear acoustics in monatomic and diatomic gases for varying values of $K n$.
\end{abstract}

\section{INTRODUCTION}

For high amplitude sound propagation at atmospheric conditions, continuum equations such as the nonlinear Euler and Navier-Stokes equations have been widely used to understand the physical properties of sound due to nonlinearity[1][2]. However, an overview of such approaches reveals that nonlinear effects are often treated separately, and then superimposed to produce a final result. Instead of employing this technique, a method such as DSMC can model all nonlinear and viscous effects in the same method. Various DSMC acoustic simulations have been compared to solutions to the linear and nonlinear Euler equations for monatomic gases to study effects such as harmonic generation, absorption, wave steepening, and nonequilibrium effects in a previous paper [3].

The Knudsen number $(\mathrm{Kn})$ is defined to be the mean free path divided by a characteristic length scale [4]. In this study, the characteristic length scale is the acoustic wavelength of the sound wave. Therefore, the Knudsen number is large for sound propagation in very dilute gases, in micro-channels, or at high frequencies, and thus requires a particle method (or kinetic theory) solution. There have been numerous attempts to study sound propagation for high $K n$ based on kinetic theory. Most of these attempts use approximations to the Boltzmann collision integral. For example, Wang Chang and Uhlenbeck [5] studied sound propagation using the method of eigenfunctions. Numerical procedures applied to the eigenfunction method were performed by Pekeris et al [6]. In Sirovich and Thurber [7], sound propagation was studied using a kinetic model description based on the Bhatnagar - Gross Krook (BGK) equation [8], while Marques [9] replaced the collision integral with a relaxation-time term. All attempts report that the speed and absorption of sound depend heavily on $\mathrm{Kn}$. Experimental results by Greenspan [10], Meyer and Sessler [11], and Schotter [12] show close agreement for the numerical methods above for Kn<1, but for larger $\mathrm{Kn}$, the results are poor.

The Direct Simulation Monte Carlo (DSMC) $[4,13,14]$ method is a particle method that was chosen for the current study. DSMC describes gas flows through direct physical modeling of particle motions and collisions. DSMC was originally designed for aerospace applications in dilute gases, and thus has been thoroughly tested for high $K n$ flows $(>0.2)$. It has been applied to hypersonic and rarefied gas flows, and has in fact become the principle method for high Kn simulations [15-18]. DSMC has also been successfully applied to modeling chemical reactions [19-21], simluating blast-structure interactions at low Kn [22], and to study sound in dilute gases (high Kn) [23]. However, there has been limited work in using DSMC for acoustics and low Kn problems (continuum regime).

The motivation for this study is three fold. First we would like to show the validity of using DSMC for low Kn applications. Second, we would like a numerical method to include all viscous and nonlinear acoustic effects, and 
third, we would like a method to accurately model sound at high $K n$. A parallel, one dimensional implementation of DSMC for monatomic and diatomic gases has been applied and compared to nonlinear Euler equation solutions as well as nonlinear acoustic theory for varying values of $K n$. There are also comparisons to experimental data.

\section{DSMC IMPLEMENTATION}

DSMC is based on the kinetic theory of gases, where representative particles are followed as they move and collide with other particles [4]. The movement of particles is determined by their velocities, while the collisions are determined statistically, but are required to satisfy mass, momentum and energy conservation. DSMC is usually limited to binary collisions. In DSMC the particle positions and velocities are initialized randomly and the boundary conditions ultimately determine the final solution, as in continuum methods.

DSMC simulations become more and more exact as the time step and the cell size tend to zero, and has in fact been proven to converge to the Boltzmann equation in the limit of small time step and small cell size [24]. It can be shown that the Boltzmann equation will reduce to the Navier-Stokes equations for $K n<0.1$ and the Euler equations for $K n->0$. Therefore, at low $K n$, this means that DSMC simulations and Euler equation solutions should give the same results. Also, the Boltzmann equation includes all physical properties of interest for sound propagation. Without modification to the method, properties such as viscous dissipation, nonlinear effects, dispersion, nonequilibrium effects, entropy waves, and more are available. In fact, DSMC has been able to model more physical phenomena than is usually contained within the Boltzmann equation, such as chemical reactions, multiple species, and diatomic particles.

In this study, acoustic waves were simulated by creating a pressure distribution in the middle of the domain, which then propagates in both directions. The shape of this pressure distribution is dependent on the acoustic source of interest. Here, Gaussian and sinusoidal pressure distributions of varying frequencies were considered. The flow velocity is initially set to 0.0 , and both monatomic and diatomic hard sphere molecules with binary elastic collisions were simulated. The cell sizes were always less than $1 / 2$ a mean free path, and care was taken in the choice of $\Delta t$ to ensure accuracy, which was computed by using a Courant or von Neumann number of 0.1 in most cases. $\Delta t$ must also be less the mean collision time. This corresponds to time step sizes in the range: $10^{-12} \leq \Delta t \leq 10^{-11}$.

\section{COMPUTATION}

An Object Oriented Programming (OOP) $[25,26]$ approach, written in $\mathrm{C}++$, is used in the development of this DSMC study. Such an implementation allows the code to be more readable, manageable, reusable, and extendable. The program is also more efficient by using such $\mathrm{C}++$ characteristics such as memory handling and pointers. Using the object oriented $\mathrm{C}++$ concepts of class structure, the code is easy to modify and understand. An object oriented technique is a very natural approach for this problem because the particles and cells are physical objects. In addition, OOP allows encapsulation, the grouping together of data and methods (i.e. functions). Using objects, the direct simulation Monte Carlo method flows in a logical sequence and is easy to understand. The responsibility of the detailed calculations is contained in the individual object.

Since the code runs independent simulations and then performs ensemble averages to reduce scatter, it lends itself very nicely to a parallel computing algorithm. Instead of having one processor do all the independent runs, it is easy to have different computers simultaneously run different ensembles, and then average them.

To achieve this simple parallel processing algorithm, a master/slave routine is constructed. The master's main job is to schedule the processors for work, organize collected data, and do the final computing of needed flow properties. The slaves compute the ensemble runs and send sampled data back to the master for final processing. The programming here is made possible by using the Message Passing Interface (MPI) [27] to send messages back and forth between processors. Also, the programming for this type of algorithm is quite simple because there are very few MPI calls.

The code was run on a 160-processor Beowulf cluster running Linux. It has a Dolphin network for fast communication and $2.8 \mathrm{GHz}$ Athlon processors. It has a peak speed of 0.4 teraflops (floating point operations per second). Despite the efficiency of the parallel algorithm, the CPU time for these DSMC programs is still fairly large 


\section{ACOUSTICS THEORY AND RESULTS}

For a sound wave with high amplitude, the linear wave equation cannot accurately simulate wave propagation. Nonlinear acoustic effects will therefore become more evident as the amplitude becomes large. Some of the major nonlinear acoustic effects are wave steepening, shock formation, harmonic generation, and dissipation [1, 2]. The speed of sound for a perfect gas is given to be:

$$
c=\sqrt{\gamma R T}
$$

which will be different for monatomic and diatomic gases. Because of the large acoustic pressures in high amplitude sound, pressure peaks travel faster than pressure troughs. An implication of this is that the wave steepens with increasing time and propagation distance.

Steepening continues until the sound wave begins to shock. This happens at a distance $\bar{x}$ which is defined to be:

$$
\bar{x}=\frac{\rho_{0} c_{0}{ }^{2}}{\beta k P_{0}}
$$

Where $\rho_{0}$ is the ambient density, $c_{0}$ is the ambient sound speed, $k$ is the acoustic wave number, $P_{0}$ is the initial acoustic pressure amplitude, and $\beta$ is the coefficient of nonlinearity, which has the value of $(\gamma+1) / 2$. This gives $\beta=4 / 3$ in a monatomic gas and $\beta=1.2$ in a diatomic gas. For low frequency, low amplitude sound waves, this shock distance is typically very large, where the signal will dissipate before the wave is given a chance to shock. However, for high frequency, high amplitude sound waves, a shock wave can develop within a few wavelengths. For sound in diatomic gases, Equation (2) implies that the shock distance will be greater than the same frequency in a monatomic gas.

DSMC simulations were implemented in monatomic and diatomic gases to investigate nonlinear and viscous effects. The diatomic routine uses the phenomenological Larsen-Borgnakke method $[4,28]$ to compute internal energy. Results for a DSMC simulation of a Gaussian pulse in Oxygen are shown in Figure 1 after it has propagated some distance. Notice that the Gaussian pulse has steepened significantly and the signal has become distorted due to nonlinearity. Figure 2 also shows the differences between monatomic simulation in Argon and a diatomic simulation in Oxygen. It appears that the diatomic case has not steepened as much as the monatomic case which we would expect since the shock distance is further for Oxygen.

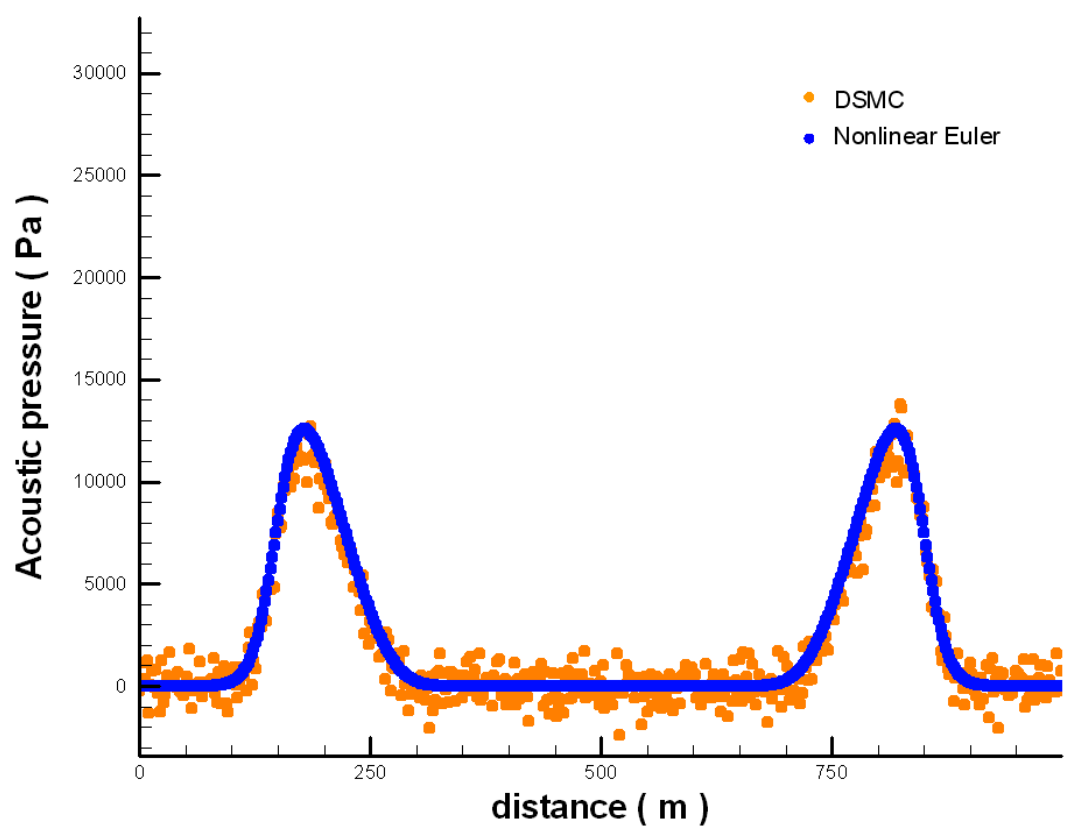

FIGURE 1. Comparisons of DSMC results in Oxygen against the nonlinear Euler equations. 


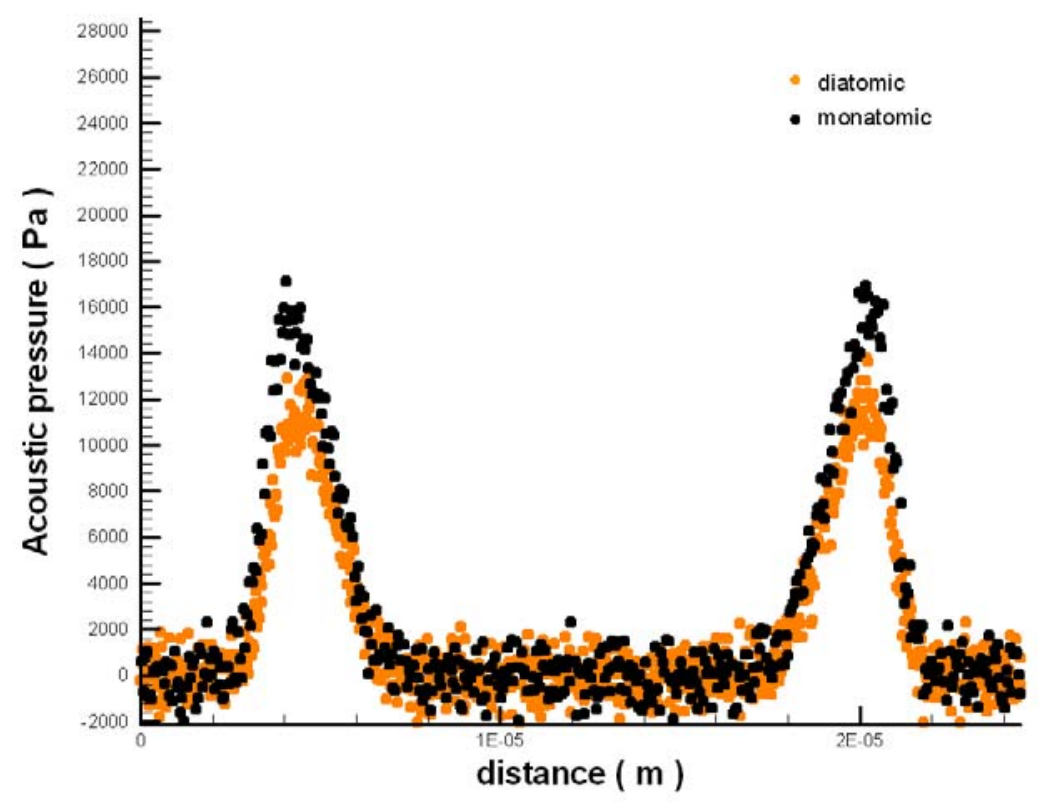

FIGURE 2. Comparisons of DSMC results in monatomic and diatomic gases

The DSMC results were compared to a finite difference solution of the nonlinear Euler equations that uses a time accurate McCormack scheme and added artificial viscosity to reduce numerical oscillations. The Gaussian pulse is 100 cells wide with each cell being $1 / 2$ of a mean-free-path wide. The mean-free-path in these conditions is approximately 50 nanometers. The domain is about 1000 cells long. Each cell has about 150 particles. There were 4000 time steps, each of size 7.5 picoseconds. The acoustic pressure amplitude of the Gaussian source is equivalent to $190 \mathrm{~dB}$, with acoustic flow velocity equal to zero. Here, $K n=0.02$. It is clear that the comparison between DSMC and nonlinear Euler solution in this case gives excellent results.

Due to wave steepening, energy from the fundamental frequency gets shifted to the higher harmonics, allowing the harmonics amplitudes to grow and thus distorting the signal. The harmonic component amplitude can be determined analytically for a sine wave in a lossless medium by the Fubini solution $[1,2]$ and the Blackstock Bridging function [1]. DSMC results for a monatomic gas are compare to the Blackstock bridging function in Reference [3]. The Fourier component amplitude of the first 4 harmonics of a $30 \mathrm{MHz}$ DSMC sine wave were compared to the analytic Blackstock Bridging function. The agreement was excellent.

\section{Rarefied Gas Acoustics}

Experimental and other kinetic theory results suggest that the absorption of sound for high $K n$ differs significantly from that for low $K n$ [5-7, 9, 29]. The scaled absorption coefficient, $\alpha / k_{0}$, where $\alpha$ is the absorption coefficient and $k_{0}$ is the acoustic wave number, for $K n$ ranging from 0.04 to 35 is shown in Figure 3 for both monatomic and diatomic cases. DSMC simulation results are compared to a classical Navier-Stokes prediction, experimental work by Greenspan [10], and previous kinetic theory results by Hadjiconstantinou and Garcia [23] whose work was based on standing waves in waveguides. The variation in $K n$ was obtained by keeping the cells per wavelength constant at 100 , but varying the cell size from $1 / 2$ of a mean free path to 3/1000 of a mean free path. The absorption coefficient results were obtained by finding the maximum pressure at each time step and calculating a line of best fit on a logarithmic scale. 


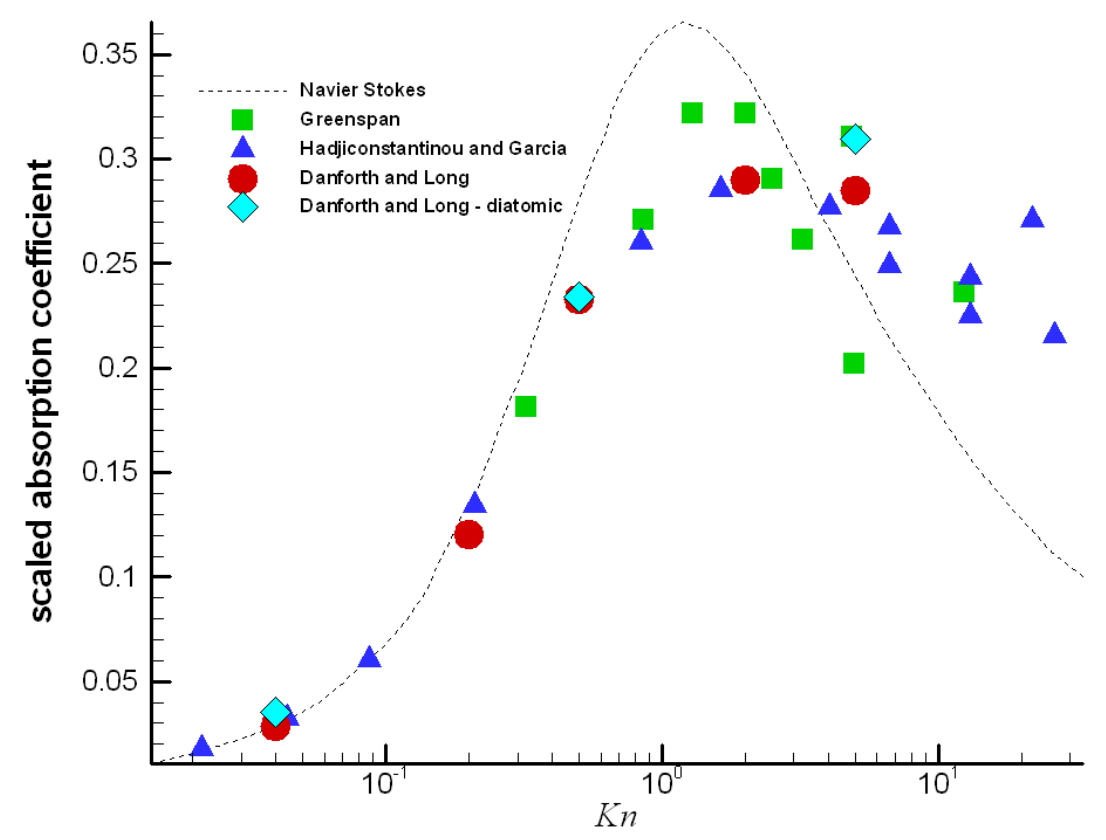

FIGURE 3. Scaled absorption coefficient for varying Kn ranging from 0.04 to 35

For high $K n$ simulations, the wavelength of the signal is significantly shorter than a mean free path. Experimental results for $K n>5.5$ are thus difficult to obtain because of scattering and high absorption. The simulations presented here have successfully captured the physics for such cases by decreasing our cell size to 3/1000 a mean free path. The calculations confirm that the Navier-Stokes prediction for the absorption coefficient is only correct for low $K n$, and a kinetic theory approach must be used for high $K n$ calculations. The absorption coefficient results are in good agreement with experimental results and previous work on standing waves.

High $\mathrm{Kn}$ acoustics can also involve nonequilibrium effects, which is another reason a kinetic theory approach is needed to study sound at high $K n$. Figures 4 and 5 show the velocity distribution functions for $K n=0.02$ and 5 in Oxygen. Both are compared to the Maxwellian distribution, and both are taken at the pressure peaks of the sound wave. For $K n=0.02$, the distribution fits the Maxwellian very well, as we would expect this system to be at equilibrium. However, for $K n=5$, there is a shift from equilibrium, as noticed in Figure 5 .

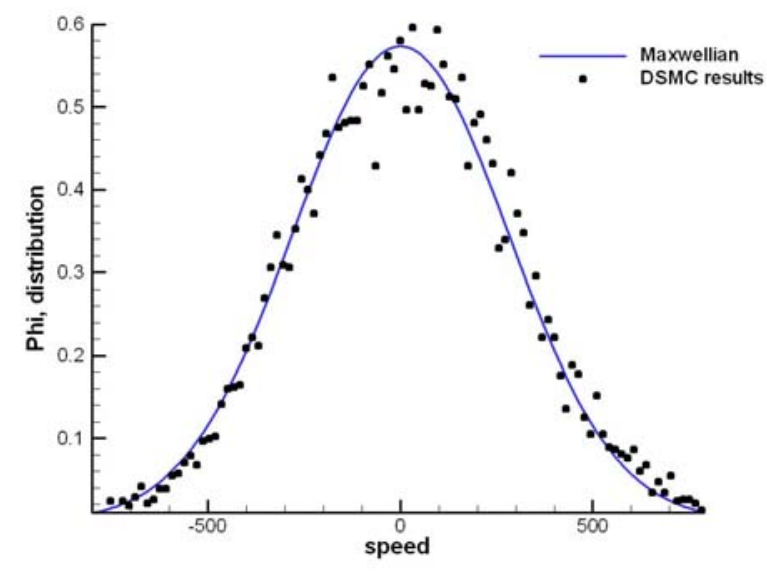

FIGURE 4. Velocity distribution curves at $K n=0.02$

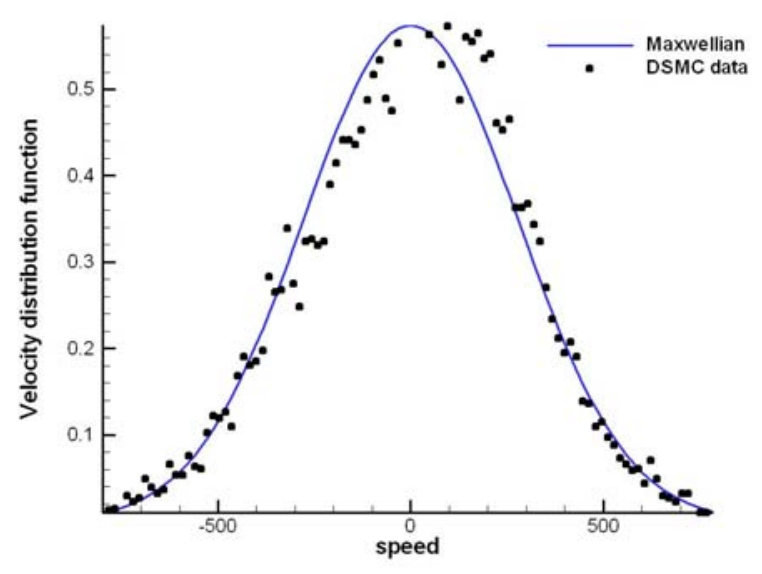

FIGURE 5. Velocity distribution curves at $K n=5$ 


\section{CONCLUSIONS}

The current study uses DSMC to study nonlinear acoustic propagation. This model was implemented using object oriented programming in $\mathrm{C}++$ which makes the entire code easier to read, maintain, and expand. The code also uses a parallel processing algorithm to further improve the efficiency of the model. Using DSMC for the study of nonlinear acoustics was validated by comparisons to the Euler equations, the Navier-Stokes equations, and experiment. Sound propagation for high $K n$ is discussed where the absorption of sound is higher than a NavierStokes or Euler prediction. Good agreement is found between experiment and previous work. Results indicate that DSMC is a suitable technique for modeling sound propagation for all values of $K n$ for both monatomic and diatomic gases.

\section{ACKNOWLEDGMENTS}

We would like to thank the National Science Foundation for funding the Consortium for Education in Many Body Applications, CEMBA (http://www.cemba.psu.edu), grant no. NSF-DGE-9987589.

\section{REFERENCES}

1. Nonlinear Acoustics, edited by Hamilton, M. and Blackstock, D., (Academic Press, San Diego, 1998).

2. Pierce, A. D., Acoustics, an Introduction to Its Physical Principles and Applications, (McGraw-Hill, New York, 1981).

3. Danforth, A. L. and Long, L. N., Journal of the Acoustical Society of America (to appear 2004).

4. Bird, G. A., Molecular Gas Dynamics and the Direct Simulation of Gas Flows, (Clarendon Press, Oxford, 1994).

5. Chang, C. S. W. and Uhlenbeck, G. E., "The Kinetic Theory of Gases" in Studies in Statistical Mechanics, edited by J. d. Boer and G. E. Uhlenbeck, North-Holland, Amsterdam, 1970, 43-75.

6. Pekeris, C. L., Alterman, Z., Finkelstein, L. and Frankowski, K., Physics of Fluids V, 1608-1616, (1962).

7. Sirovich, L. and Thurber, J. K., Journal of the Acoustical Society of America 37, 329, (1965).

8. Bhatnagar, P. L., Gross, E. P. and Krook, M., Physical Review 94, 511-525, (1954).

9. Marques, W., Journal of the Acoustical Society of America 106, 3282-3288, (1999).

10. Greenspan, M., Journal of the Acoustical Society of America 28, 644, (1956).

11. Meyer, E. and Sessler, G., Z. Physics 159, 15-39, (1957).

12. Schotter, R., Physics of Fluids 17, 1163-1168, (1974).

13. Alexander, F. J. and Garcia, A. L., Computers in Physics 11, 588, (1997).

14. Oran, E. S., Oh, C. K. and Cybyk, B. Z., Annual Review of Fluid Mechanics 30, 403-440, (1998).

15. Bird, G. A., Physics of Fluids 13, 2676, (1970).

16. Bird, G. A., Annual Review of Fluid Mechanics 11, 11-31, (1978).

17. Muntz, E. P., Annual Review of Fluid Mechanics 21, 387-417, (1989).

18. Long, L. N., AIAA Journal 29, (1991).

19. Anderson, J. B. and Long, L. N., Journal of Chemical Physics 118, 3102-3110, (2003).

20. Long, L. N. and Anderson, J. B., The simulation of detonations using a Monte Carlo Method in 22rd International Symposium on Rarefied Gas Dynamics Conference, (2000).

21. O'Connor, P. D., Long, L. N. and Anderson, J. B., Direct Simulation of Ultrafast Detonations in Mixtures in 24th International Symposium on Rarified Gas Dynamics Conference, (2004).

22. Sharma, A. and Long, L. N., Journal of Computational Physics (to appear 2004).

23. Hadjiconstantinou, N. G. and Garcia, A. L., Physics of Fluids 13, 1040-1046, (2001).

24. Wagner, W., Journal of Statistical Physics 66, 1011, (1992).

25. Yang, D., C++ and Object-Oriented Numeric Computing, (Springer, New York, 2001).

26. Shtern, V., Core C++, (Prentice Hall PTR, Upper Saddle River, 2000).

27. Pacheco, P. S., Parallel Programming with MPI, (Morgan Kaufmann Publishers, San Fransisco, 1997).

28. Borgnakke, C. and Larson, P. S., Journal of Chemical Physics 18, 405-420, (1975).

29. Greenspan, M., "Transmission of Sound Waves in Gases at Very Low Pressures" in Physical Acoustics, edited by W. P. Mason, Academic Press, New York, 1965, 1-45. 